\title{
ISOMORPHIC FACTORISATIONS V: DIRECTED GRAPHS
}

\author{
FRANK HARARY, ROBERT W. ROBINSON AND NICHOLAS C. WORMALD
}

\begin{abstract}
An isomorphic factorisation of a digraph $D$ is a partition of its arcs into mutually isomorphic subgraphs. If such a factorisation of $D$ into exactly $t$ parts exists, then $t$ must divide the number of arcs in $D$. This is called the divisibility condition. It is shown conversely that the divisibility condition ensures the existence of an isomorphic factorisation into $t$ parts in the case of any complete digraph. The sufficiency of the divisibility condition is also investigated for complete $m$-partite digraphs. It is shown to suffice when $m=2$ and $t$ is odd, but counterexamples are provided when $m=2$ and $t$ is even, and when $m=3$ and either $t=2$ or $t$ is odd.
\end{abstract}

\$1. Introduction. An isomorphic factorisation of a digraph $D=(V, E)$ is a partition $\left\{E_{1}, E_{2}, \ldots, E_{t}\right\}$ of the arc set $E$ such that the spanning sub-digraphs $\left(V, E_{1}\right),\left(V, E_{2}\right), \ldots$, $\left(V, E_{t}\right)$ are all isomorphic. If $H \simeq\left(V, E_{1}\right)$ we say $H$ divides $D$ and write either $H \mid D$ or $H \in D / t$. Also, if $D$ has an isomorphic factorisation into exactly $t$ isomorphic subgraphs, we say that $D$ is divisible by $t$ and write $t \mid D$. Similarly, an isomorphic factorisation of an ordinary (symmetric) graph $G$ is defined in [4] to be a partition of its line set which induces isomorphic subgraphs, and the above notation is used in the analogous fashion for graphs.

For given $t$ and a given digraph $D$ having exactly $q$ arcs, an obvious necessary condition for the divisibility of $D$ by $t$ is that $t$ divide $q$. This is called the Divisibility Condition for $D$ and $t$. To avoid triviality we always take $t>1$. All basic graph theoretic notation can be found in [2].

In the first paper in this series [4] we showed that whenever $G$ is a complete graph the Divisibility Condition is sufficient for the divisibility of $G$ by $t$. The third paper [5] examined analogous questions for complete $r$-partite graphs when $r>1$. It was found that when $r$ is 2 the Divisibility Condition is sufficient, but when $r$ is 3 and $t$ is odd the Divisibility Condition is insufficient for the divisibility of a complete $r$-partite graph. Our present objective is to examine complete digraphs and complete multipartite digraphs in the same way.

Notation. If $G$ is a graph, then $D G$ is the digraph of $G$, where $D G$ has a symmetric pair of arcs for each line of $G$.

In the next section we show the sufficiency of the Divisibility Condition for the complete digraph $D K_{p}$ on $p$ points, for all $p$. In fact, for given $p$ and $t$, the Divisibility Condition implies the existence of a self-converse digraph in $D K_{p} / t$. Complete $r$-partite digraphs are examined in Section 3. We show that for $r=2$ the Divisibility Condition is always sufficient when $t$ is odd, but examples of insufficiency exist for all even $t$. When $r=3$, however, we show that the Divisibility Condition is not sufficient for any odd $t$ or for $t=2$. This insufficiency when $t=2$ is also shown to apply for any $r$ which is divisible by 4 . In the final section related problems, results and conjectures are considered.

§2. Complete Directed Graphs. Our purpose in this section is to prove that the Divisibility Condition is sufficient for the existence of a self-converse digraph in 
$D K_{p} / t$ for all $p$ and $t$. When $t$ is odd this result is a corollary of the Divisibility Theorem for complete graphs [4], whilst the case when $t$ is even is handled by modifying the arguments used to prove the Divisibility Theorem.

THEOREM 1. If $t$ divides $p(p-1)$ then the complete directed graph $D K_{p}$ is divisible by $t$. In fact, $D K_{p} / t$ contains a self-converse digraph.

Proof. Five cases will be considered, mainly because the general constructions employed are not applicable for small values of $p$ and $t$.

Case 1. $t$ is odd. It follows that $t \mid p(p-1) / 2$, so by the Divisibility Theorem of [4], the complete graph $K_{p}$ is divisible by $t$. If $G$ is a graph in $K_{p} / t$, then the digraph of $G$, namely $D G$, is a self-converse member of $D K_{p} / t$.

Case 2. $t=2$. Consider the complete directed graph $D K_{p}$ with point set $\{1,2, \ldots, p\}$. Let $D$ be the digraph containing exactly the arcs $(i, j)$ for which $i<j$. Then $D$ is self-converse and is in $D K_{p} / 2$. (This $D$ is the transitive tournament which is denoted by $T_{p}$ in Moon [8].)

Case 3. $t=4$. In this case the Divisibility Condition implies that $p=m$ or $m+1$ for $m$ divisible by 4 . We give first the construction for $p=m$, then show how to modify it for $p=m+1$.

Given $m=4 s$ let $A_{1}, A_{2}, A_{3}$ and $A_{4}$ be four disjoint point sets each of cardinality $s$, and consider the complete directed graph $D K_{m}$ with point set

$$
V=A_{1} \cup A_{2} \cup A_{3} \cup A_{4} \text {. }
$$

Let $D_{0}$ be the digraph with point set $V$ containing just those arcs $(u, v)$ for which $u \in A_{1}$ and $v \in A_{2}, u \in A_{1}$ and $v \in A_{3}, u \in A_{3}$ and $v \in A_{2}$, or $u, v \in A_{4}$. Then $D_{0}$ is self-converse and is a member of $D K_{m} / 4$. A factorisation of $D K_{m}$ into four digraphs isomorphic to $D_{0}$ can be obtained as follows. Let $\phi$ be an automorphism of the point
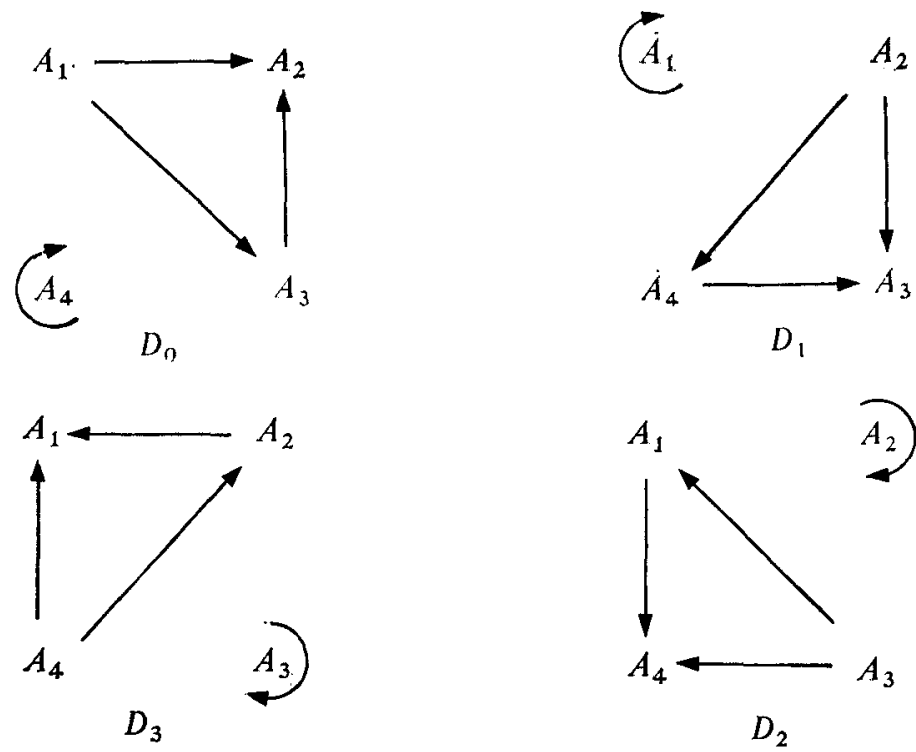

Fig 1. An isomorphic factorisation of $D K_{m} / 4$ when $m=4 \mathrm{~s}$ 
set of $D K_{m}$ such that $\phi\left(A_{1}\right)=A_{2}, \phi\left(A_{2}\right)=A_{3}, \phi\left(A_{3}\right)=A_{4}$ and $\phi\left(A_{4}\right)=A_{1}$. Then for $i=1,2$ and 3, the map $\phi^{i}$ provides an isomorphism between $D_{0}$ and another digraph, say $D_{i}$. The digraphs $D_{0}, D_{1}, D_{2}$ and $D_{3}$ provide the required factorisation, as illustrated in Figure 1.

Next, let $w$ be a point not in $V$ and consider the complete directed graph $D K_{m+1}$ with point set $V^{\prime}=V \cup\{w\}$. Let $D_{0}{ }^{\prime}$ be the digraph with point set $V^{\prime}$ containing those arcs in $D_{0}$ together with all arcs $(u, w)$ and $(w, u)$ for which $u \in A_{4}$. It follows that $D_{0}{ }^{\prime}$ is self-converse, and is an element of $D K_{m+1} / 4$. An isomorphic factorisation of $D K_{m+1}$ can be found in the same way as for $D K_{m}$, this time using the automorphism of $V^{\prime}$ which agrees with $\phi$ on $V$ and fixes $w$.

Case 4. $t>4$ is even and $p \leqslant 4$. It follows that $(p, t)=(3,6),(4,6)$ or $(4,12)$. It is easily seen that in these cases $D K_{p} / t$ contains respectively a single arc, a symmetric pair of arcs, and a single arc (plus isolates).

Case 5. $t>4$ is even and $p \geqslant 5$. We use induction on $p$. First, suppose $p \leqslant t-1$. Then since $t \mid p(p-1)$ and $t>2$ we have $p \leqslant t-2$. It follows that

$$
\frac{p(p-1)}{2 t}+\frac{1}{2}<\frac{p-1}{2}
$$

So if $m=p(p-1) / t$, we see that $(m+1) / 2<(p-1) / 2$. Therefore, by Lemma 2 of [4], the complete graph $K_{p}$ can be line-labelled so that any $\frac{1}{2}(m+1) / 2$ consecutive lines are disjoint, where $x$ denotes the largest integer not exceeding $x$. Using this line-labelling of $K_{p}$, we label the arcs of $D K_{p}$ with the integers $1,2, \ldots, p(p-1)$ by assigning the numbers $2 i-1$ and $2 i$ to the two arcs which correspond to the line labelled $i$. Any $m$ consecutive arcs will then induce a spanning sub-digraph of $D K_{p}$ isomorphic to $(m / 2) D K_{2}$ when $m$ is even, and $\frac{1}{2}(m-1) D K_{2}$ together with an isolated arc when $m$ is odd. Hence $D K_{p}$ is divisible by $t$, and in both cases the factor digraph is self-converse.

It can now be assumed that $p \geqslant t$ and that $D K_{p-t} / t$ contains a self-converse digraph $D$. In the event that $p=t$, the empty digraph will suffice for $D$. By using similar methods to the proof of Lemma 3 in [4], we will construct a digraph in $D K_{p} / t$ with point set $\{1,2, \ldots, p\}$. First, take an isomorphic factorisation $D_{1}, D_{2}, \ldots, D_{t}$ of $D K_{p-t}$ on the point set $\{t+1, t+2, \ldots, p\}$ with $D_{i} \cong D$ for all $i$. To $D_{1}$ add the points $\{1,2, \ldots, t\}$, add all arcs between the points of $D_{1}$ and the point 1 , and add the symmetric pairs of arcs with endpoints $\{1,2\},\{1,3\}, \ldots,\{1,(t / 2)+1\},\{1,(t / 2)+2\}$ together with the arc $(t / 2, t)$. The resultant digraph $H_{1}$ is self-converse. This is because $t \geqslant 6$, so the arc $(t / 2, t)$ is disjoint from all other arcs, and because $D_{1}$ is self-converse. Furthermore, $H_{1}$ is an element of $D K_{p} / t$, the $i$-th digraph in an appropriate factorisation of $D K_{p}$ being obtained by permuting the points of $D_{1}$ so as to form $D_{i}$ and simultaneously applying the permutation $(12 \ldots t)^{i-1}$ to the points $\{1,2, \ldots, t\}$.

§3. Complete r-partite Directed Graphs. The complete r-partite directed graph of type $\left(n_{1}, n_{2}, \ldots, n_{r}\right)$ is the complement of the disjoint union $D K\left(n_{1}\right) \cup \ldots \cup D K\left(n_{r}\right)$ of complete directed graphs and is denoted by $D K\left(n_{1}, n_{2}, \ldots, n_{r}\right)$. As usual, the complete 2-partite directed graphs are called bipartite while the 3-partite graphs are called tripartite. In this section we examine the question: For which $r$ and $t$ are all complete $r$-partite directed graphs, satisfying the Divisibility Condition, divisible by $t$ ? We demonstrate that for $r=2$, this is true, if, and only if, $t$ is odd. For $r=3$, on 
the other hand, we know of no value of $t$ such that all complete tripartite directed graphs satisfying the Divisibility Condition are divisible by $t$. For $t=2$ and for all odd $t$ we give examples of complete tripartite digraphs satisfying the Divisibility Condition but not divisible by $t$.

THEOREM 2. The Divisibility Condition is sufficient for the existence of a digraph in $D K(m, n) / t$ for all $m$ and $n$, if, and only if, $t$ is odd.

Proof. Suppose $t$ is odd. Then $t \mid 2 m n$ implies $t \mid m n$, so by Theorem 1 of [5], the complete bipartite graph $K_{m, n}$ is divisible by $t$. Thus $G \in K_{m, n} / t$ for some graph $G$, and its digraph $D G$ is therefore a member of $D K(m, n) / t$.

Suppose on the other hand that $t$ is even. We shall show that if $m=s t / 2$ where $s \geqslant 3$ is odd, the digraph $D K(m, 1)$ is not divisible by $t$ even though the Divisibility Condition is satisfied. Suppose to the contrary that there is some $D \in D K(m, 1) / t$. It follows that $D$ has precisely $s$ arcs. As a consequence of the structure of $D K(m, 1)$, these arcs must all be adjacent at one point, $u$ say. As $s \geqslant 3$, the point $u$ either has indegree greater than 1 or out-degree greater than 1 . Therefore, in any subdigraph of $D K(m, 1)$ isomorphic to $D$, the point $u$ of $D$ must coincide with the point of in degree $m$ in $D K(m, 1)$. So if $u$ has in-degree $k$ in $D$, it follows that $m=t k$. This contradicts the choice of $m=s t / 2$ where $s$ is odd.

We next consider complete tripartite directed graphs. It was shown in [5] that if $t>1$ is odd and $m \geqslant t(t+1)$, the complete tripartite graph $K(1,1, m)$ is not divisible by 3 . This was done by considering the degrees of the points in a hypothetical member of $K(1,1, m) / t$. If out-degrees are considered instead, the same argument suffices to prove the following.

THEOREM 3. If $t>1$ is odd and $m \geqslant t(t+1)$, then $D K(1,1, m) / t$ is empty.

For $r=3$ and $t$ even, we do not have a result as broad as Theorem 3; all we have is the following counterexample to the sufficiency of the Divisibility Condition when $t=2$.

THEOREM 4. If $m>5$ is odd, then $D K(1,2, m) / 2$ is empty.

Proof. Assume $m>5$ is odd and suppose $D K(1,2, m) / 2$ is nonempty. In $D=D K(1,2, m)$ let $a$ be the point of degree $m+2, b_{1}$ and $b_{2}$ the points of degree $m+1$, and $c_{1}, \ldots, c_{m}$ the points of degree 3 . Let $D^{\prime}$ and $D^{\prime \prime}$ denote the two spanning subdigraphs of an isomorphic factorisation of $D$ into two parts. For any point $u$ of $D$, let $u^{\prime}$ denote the out degree of $u$ in $D^{\prime}$, and $u^{\prime \prime}$ the out-degree of $u$ in $D^{\prime \prime}$. Also, let $\phi$ be a permutation of the points of $D$ which maps $D^{\prime}$ to $D^{\prime \prime}$.

We first note that $a^{\prime}+a^{\prime \prime}=m+2$. Since $m$ is odd, this means either

$$
a^{\prime}>(m+2) / 2 \quad \text { or } \quad a^{\prime \prime}>(m+2) / 2 \text {; }
$$

we can assume $a^{\prime}>(m+2) / 2$, if necessary interchanging $D^{\prime}$ and $D^{\prime \prime}$. Now, $\phi(a)$ cannot be $a$ because $a^{\prime} \neq a^{\prime \prime}$, and $\phi(a)$ cannot be $c_{i}$ for any $i$ because $c_{i}^{\prime \prime} \leqslant 3$ whereas $a^{\prime}>3$ since $m>5$. Thus $\phi(a)=b_{1}$ or $b_{2}$; take $\phi(a)=b_{1}$, if necessary interchanging $b_{1}$ and $b_{2}$. It follows that $b_{1}{ }^{\prime \prime}=a^{\prime}$ and $b_{1}{ }^{\prime}=m+1-a^{\prime}<m / 2$.

Now suppose $b_{2}^{\prime}>(m+1) / 2$. It follows that $\phi\left(b_{2}\right)^{\prime}>(m+1) / 2>3$, so $\phi\left(b_{2}\right)=a$ or $b_{2}$. But $a^{\prime \prime}=m+2-a^{\prime}<(m+2) / 2$, and thus $a^{\prime \prime} \leqslant(m+1) / 2$, which disallows $\phi\left(b_{2}\right)=a$. Hence $\phi\left(b_{2}\right)=b_{2}$, so $b_{2}{ }^{\prime}=b_{2}{ }^{\prime \prime}$, which contradicts 
$b_{2}{ }^{\prime}>(m+1) / 2$. On the other hand, if $b_{2}{ }^{\prime}<(m+1) / 2$, it follows that $b_{2}{ }^{\prime \prime}>$ $(m+1) / 2$, and it can be seen that $\phi^{-1}\left(b_{2}\right)$ must consequently be $b_{1}$. Thus $b_{1}{ }^{\prime}=b_{2}{ }^{\prime \prime}>$ $(m+1) / 2$, which contradicts $b_{1}{ }^{\prime}<m / 2$. We must conclude that $b_{2}{ }^{\prime}=b_{2}{ }^{\prime \prime}=(m+1) / 2$. Moreover, this means that $\phi^{-1}\left(b_{2}\right)$ can only be $b_{2}$, as $a^{\prime}>(m+2) / 2$ and $b_{1}{ }^{\prime}<m / 2$, while $c_{1}{ }^{\prime} \leqslant 3<(m+1) / 2$. Since $\phi\left(b_{1}\right) \neq a$, we therefore have $\phi^{-1}(a)=c_{j}$ and $\phi\left(b_{1}\right)=c_{k}$ for some $j$ and $k$. As $\phi$ fixes $b_{2}, \phi\left(c_{k}\right)$ is either $a$ (in which case $j=k$ ) or $c_{l}$ for some $l \neq k$. We consider these two possibilities separately, and reach a contradiction in each case.

Case 1. $\phi\left(c_{k}\right)=a$. It follows that the permutation $\phi$ contains the 3-cycle $\left(a b_{1} c_{k}\right)$. If the arc $\left(a, b_{1}\right)$ is in $D^{\prime}$, it follows that $\left(b_{1}, c_{k}\right) \in D^{\prime \prime}$, so $\left(b_{1}, c_{k}\right) \notin D^{\prime}$, and following on from this we have $\left(c_{k}, a\right) \notin D^{\prime \prime},\left(c_{k}, a\right) \in D^{\prime},\left(a, b_{1}\right) \in D^{\prime \prime}$ and thus $\left(a, b_{1}\right) \notin D^{\prime}$. A similar contradiction is reached if $\left(a, b_{1}\right) \in D^{\prime \prime}$.

Case 2. $\phi\left(c_{k}\right)=c_{l}$. It follows that $\phi^{-1}\left(c_{j}\right)=c_{h}$ for some $h \neq j$. Then $D$ does not contain the arc $\left(c_{h}, c_{j}\right)$, so in particular, neither does $D^{\prime}$. Using the same type of argument as in Case 1 , we find therefore that $D^{\prime}$ contains $\left(c_{j}, a\right), D^{\prime \prime}$ contains $\left(a, b_{1}\right)$, $D^{\prime}$ contains $\left(b_{1}, c_{k}\right)$ and hence $D^{\prime \prime}$ contains $\left(c_{k}, c_{l}\right)$ which is a contradiction.

We conclude that $D / 2$ is empty for odd $m>5$, which proves the theorem.

Another result shown in [5] was that, if $4 \mid(r+1)$ and $m>r+1$, then the complete $(r+1)$-partite graph $K(r: 1, m)$ is not divisible by 2 , where $K(r: 1, m)$ has $r$ parts consisting of a single point and one part containing $m$ points. As in the case of Theorem 3, the following corresponding result for directed graphs is obtained by considering out-degrees rather than degrees in the proof.

THEOREM 5. Let $D K(r: 1, m)$ be the complete $(r+1)$-partite directed graph with $r$ parts of cardinality 1 and one part of cardinality $m$. If $4 \mid(r+1)$ and $m>r+1$, then $D K(r: 1, m)$ is not divisible by 2 .

§4. Related Problems. We have been studying various complete $r$-partite directed graphs and different values of $t$ in an attempt to find precisely when the Divisibility Condition is sufficient to ensure the existence of an isomorphic factorisation. Sufficiency was shown for all complete directed graphs with any $t$, and for all complete bipartite directed graphs with any odd $t$. Counterexamples to sufficiency were found with $r=2$ for any even $t$, and with $r=3$ for any odd $t>1$ and for $t=2$. We conjecture that for any $t>1$, there is a complete tripartite directed graph $D$ such that $D$ and $t$ satisfy the Divisibility Condition but $D / t$ is empty.

One can of course consider the sufficiency of the Divisibility Condition for more general classes of digraphs, as for example the symmetric digraphs. Each symmetric digraph is of the form $D G$ for some graph $G$. The number of arcs in $D G$ is even, and we can ask for which graphs $G$ does 2 actually divide $D G$ ? A survey of the graphs on $p \leqslant 5$ points reveals that $D G / 2$ is nonempty for all such graphs except when $G$ is isomorphic to $K_{1,3}$ or to the graph $H$ in Figure 2. Of course $D K_{1,3}$ is one of the

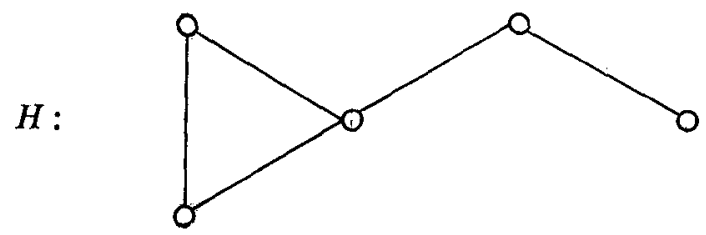

Fig. 2 A graph $H$ with $D \mathrm{H} / 2$ empty 
counterexamples provided in the proof of Theorem 2, but the other graph, $H$, is not. The general question appears to be difficult.

A number of authors have approached isomorphic factorisations of complete digraphs and complete bipartite digraphs as problems in combinatorial designs. A survey of recent results is given by Bermond and Sotteau [1], and a detailed translation of the language of block designs into the language of graphs and their factors is given in [6]. The most general results are due to Wilson [9], who showed that for every digraph $D$ there is a lower bound $N_{0}(D)$ such that $D \mid D K_{p}$ for all $p \geqslant N_{0}(D)$ satisfying certain obvious necessary conditions. In special cases more is known aboul $N_{0}(D)$. For example $N_{0}(D)$ is determined in [3] to be 0 when $D$ is any oriented 4-cycle, and Köhler [7] has shown for the directed 6-cycle $Z_{6}$ that when $p \neq 12$, $Z_{6} \mid D K_{p}$, if, and only if, $p>6$ and $p \equiv 0,1,3$ or $4(\bmod 6)$. In the latter, it is alsc shown that $Z_{2 k} \mid D K_{p}$ if $p \equiv 1(\bmod 2 k)$ and that $Z_{2 k} \mid D K_{m, n}$ if $k \mid m$ and $n \geqslant k$.

What distinguishes the factorisation problem considered in the present series of papers is that the number of parts $t$ for the factorisation is held constant. Studies motivated by design theory generally hold the factor itself constant. There is also an intermediate class of problems in which the number of factors increases linearly with the number of points in the graph to be factored. Such is the case with factorisations of complete graphs into spanning subgraphs which are unions of cycles; when the cycles are all of the same length such a factorisation is called a resolvable design (see [6]). The tantalising open problem of P. J. Kelly also falls into this intermediate class. He conjectured [8, p. 7] that if $T$ is any regular tournament on $p$ points then $Z_{p} \mid T$. This requires a factorisation of $T$ into exactly $(p-1) / 2$ copies of $Z_{p}$.

We conclude by conjecturing that the Divisibility Condition does suffice for the family of complete multipartite digraphs of the form $D K(m, m, \ldots, m)$, analogous to the conjecture for graphs in [5].

\section{References}

1. J. C. Bermond and D. Sotteau. "Graph decompositions and G-designs", Proc. 5th British Combinatorial Conf., C. Nash-Williams and J. Sheehan, eds. (Utilitas, Winnipeg, 1976), 53-72.

2. F. Harary. Graph Theory (Addison-Wesley, Reading, Mass., 1969).

3. F. Harary, K. Heinrich and W. D. Wallis. "Decomposition of complete symmetric digraphs into the four oriented quadrilaterals ", Proc. Int. Conf. Combinatorial Theory, D. Holton and J. Seberry, eds. Springer Notes, $686(1978), 165-173$.

4. F. Harary, R. W. Robinson and N. C. Wormald. "Isomorphic factorisations I: Complete graphs ", Trans. Amer. Math. Soc., 242 (1978), 243-260.

5. F. Harary, R. W. Robinson and N. C. Wormald. "Isomorphic factorisations III: Complete multipartite graphs", Proc. Int. Conf. Combinatorial Theory D. Holton and J. Seberry, eds. Springer Notes, 686 (1978), 47-54.

6. F. Harary and W. D. Wallis. "Isomorphic Factorisations II: Combinatorial designs ", Proc. 8th Southeastern Conf. on Combinatorics, Graph Theory and Computing (Utilitas, Winnipeg, 1978), 13-28.

7. E. Köhler. "Zerlegung total gerichteter Graphen in Kreise ", Manuscripta Math., 19 (1976), 151-164.

8. J. W. Moon. Topics on Tournaments (Holt, Rinehart and Winston, New York, 1968).

9. R. M. Wilson. "Decompositions of complete graphs into subgraphs isomorphic to a given graph", Proc. 5th British Combinatorial Conf., C. Nash-Williams and J. Sheehan, eds. (Utilitas, Winnipeg, 1976), 647-659. 
Department of Mathematics, University of Michigan,

Ann Arbor, Michigan, 48109, U.S.A.

Department of Mathematics, University of Newcastle, New South Wales, 2308, Australia.
05C20: COMBINATORICS;

Graph

theory;

Received on the 16th of February, 1978. 